\title{
Use of the KineSpring system in the treatment of medial knee osteoarthritis: preliminary results
}

\author{
VINCENZO MADONNA ${ }^{1}$, VINCENZO CONDELLO ${ }^{1}$, GIANLUCA PIOVAN ${ }^{2}$, DANIELE SCREPIS ${ }^{3}$, \\ CLAUDIO ZORZI ${ }^{1}$
}

${ }^{1}$ Department of Orthopaedics, Sacro Cuore Don Calabria Hospital, Negrar (VR), Italy

2 Department of Orthopaedics, University of Trieste, Italy

3 Department of Orthopaedics, University of Perugia, Italy

\begin{abstract}
Purpose: the purpose of this study was to analyze our preliminary results obtained with the KineSpring system in patients suffering from medial compartment knee osteoarthritis (OA).

Methods: between September 2012 and May 2014, 53 patients underwent treatment with the KineSpring system. Patient self-assessment was performed preoperatively and at 3, 6 and 12 months postoperatively, and included the KOOS, Tegner activity score, Lysholm functional knee score, VAS knee pain score, and IKDC score. Device- and procedure-related adverse events were recorded.

Results: mean KOOS subscales, except for the Sport/Recreation subscale at six months, improved over time. Mean WOMAC Pain and Function domains, Lysholm score, IKDC score and VAS knee pain score improved over the follow-up period and were significantly improved at 3, 6 and 12 months postoperatively compared to baseline. Mean Tegner score improved slightly over time. In 5 of the 53 $(9.4 \%)$ patients re-operation was necessary. In $3 \mathrm{pa}-$ tients the device was removed due to infection (one case) or persistent knee pain (two cases). Surgical arthrolysis was performed in two patients.

Conclusions: in our preliminary experience, the KineSpring system gave good short-term clinical results.
\end{abstract}

\footnotetext{
Corresponding Author:

Vincenzo Madonna, MD

Orthopaedic Division, Sacro Cuore Don Calabria Hospital

Via Don A. Sempreboni 5

37024 Negrar (VR), Italy

E-mail:vmadonna@me.com
}

Level of evidence: Level IV, therapeutic case series.

Keywords: KineSpring, medial compartment, knee osteoarthritis, varus, unloading.

\section{Introduction}

Knee osteoarthritis $(\mathrm{OA})$ is a common progressive degenerative disease affecting about $13 \%$ of females and $10 \%$ of males aged 60 years and older (1). It is one of the leading causes of musculoskeletal pain and disability in the adult population. Over the last century, changes in lifestyle and an increase in physical activity across all age groups have led to a higher incidence of knee OA affecting a younger population.

Knee OA, especially in the early phase and in younger patients, is often limited to a single compartment. During normal gait, the medial compartment bears $60-80 \%$ of the load acting on the knee, and this likely predisposes this compartment to an increased incidence and faster progression of joint degeneration (2). Furthermore, when varus knee alignment is present the peak adduction moment increases, resulting in a commensurate increase in medial compartment loads $(3,4)$. Medial compartment loads are further increased following injury or meniscectomy of the medial meniscus. Medial knee OA in the middle-aged patient is one of the most challenging pathologies to treat. Often there is mild to moderate radiographic OA (Kellgren-Lawrence grade I-III), nevertheless patients typically complain that they are unable to participate in sports and recreational activities, or stand for a long time.

Non-surgical treatments such as wedged insoles, knee braces, weight loss and muscular strength training 
reduce knee joint loading and may alleviate OA symptoms; however, these treatments rarely achieve long-term control of symptoms (5). Especially when medial meniscal tears are present, which they very frequently are in this age group, arthroscopic meniscectomy is not able to improve symptoms but, conversely, may worsen them. When a moderate to severe varus deformity is present, high tibial osteotomy (HTO) is the gold standard surgical treatment. With more neutral leg alignment, unicompartmental knee arthroplasty (UKA) has been the only viable option, however in younger patients, UKA is associated with an increased risk of polyethylene wear and a lower survival rate due to higher activity demands (6).

There is a clear need for novel treatments to fill the therapeutic gap between ineffective non-surgical treatment and invasive surgical options with limited durability and safety. The ideal treatment should stop the progression of the degenerative process and alleviate the pain without altering the anatomy and biomechanics of the knee joint.

Gabriel et al. presented a new unloading device designed to fulfill these objectives (7). They described the first generation of the KineSpring ${ }^{\circledR}$ Knee Implant System (Moximed, Hayward, CA, USA), which is an extra-articular and extra-capsular load absorber. The aim of this device was to offer a minimally invasive, joint-sparing and reversible procedure. Recently, London et al. described the collective clinical experience gained from 99 patients enrolled across three clinical trials with a mean follow-up of 17 months. They concluded that KineSpring provides an effective therapeutic option for medial knee OA $(8,9)$. To date, very few clinical studies have reported results obtained with the current generation of KineSpring. The purpose of this study is to report and discuss our preliminary results obtained with the single spring KineSpring implant in 53 patients affected by medial compartment OA.

\section{Methods}

\section{Participants}

Between September 2012 and May 2014, 53 patients (45 males and 8 females) underwent treatment with the KineSpring system and are included in this analysis. They had a mean age of $54.2 \pm 6.8$ years (range,
$40.1-68.2$ years) and an average body mass index of $27.4 \pm 2.6$ (range, $22.5-32.3$ ).

All the patients underwent a thorough preoperative assessment including standard knee X-rays, axial patellar projections, weight-bearing full-length lower limb radiographs, magnetic resonance imaging, clinical examination and standard preoperative blood tests. Patients were included if they fulfilled the following criteria: 40 to 70 years of age with symptomatic, radiographically-confirmed medial knee OA resistant to nonoperative care (Fig. 1). Exclusion criteria were: symptomatic lateral or patellofemoral compartment $\mathrm{OA}$, radiographic signs of lateral or patellofemoral $\mathrm{OA}>$ grade II, varus alignment $>10$ degrees, inflammatory joint disease, moderate to severe osteoporosis, anteroposterior or varus-valgus instability, and active infection.

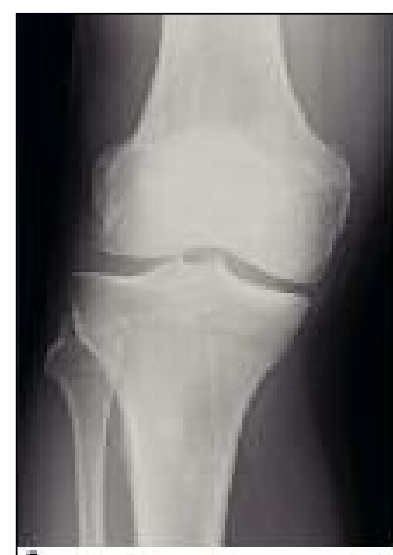

A
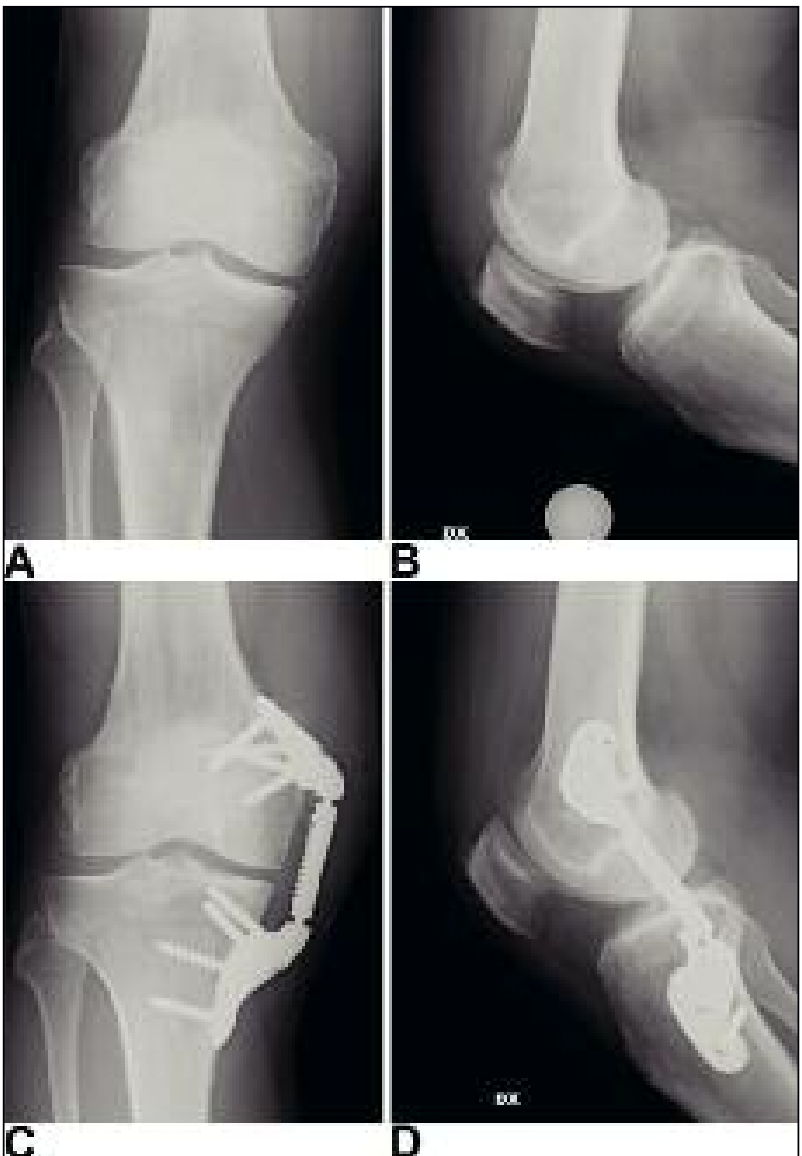
B

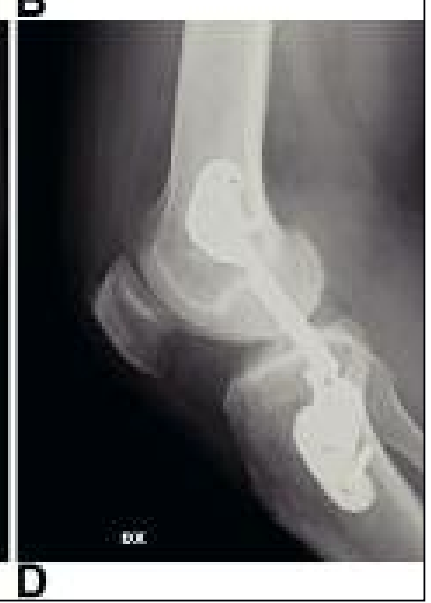

Fig. 1. Preoperative standard AP (A) and lateral (B) X-rays and oneyear postoperative X-rays (C-D). 


\section{Surgical Technique}

All surgeries were performed by the same surgeon at our institution.

The patient is placed supine with the operative leg elevated on a foot roll and the contralateral limb slightly lowered to allow unobstructed fluoroscopic view. The surgical approach to the distal femur consists of a straight skin incision, approximately $5 \mathrm{~cm}$ long, starting from the medial epicondyle and directed proximally and anteriorly. The fascia is dissected $2 \mathrm{~mm}$ from the vastus medialis obliquus muscle fibers via a standard subvastus approach and tissues are bluntly retracted exposing the periosteal tissue surrounding the medial femoral condyle. A C-arm is positioned underneath the operating table with the imaging head extending above the patient. Once a true lateral radiographic view of the distal femur is established, the femoral target position is selected under fluoroscopic guidance and a $\mathrm{K}$-wire is inserted into the target point. With the knee in full extension an alignment guide is placed over the femoral target $\mathrm{K}$-wire. The guide is then aligned parallel to the tibia long axis and at the distal end of the guide two $\mathrm{K}$-wires are inserted through the alignment guide into the tibia to define the proximal starting point of the tibial incision and alignment of the absorber. The alignment guide is removed leaving only the three $\mathrm{K}$-wires in place.

A femoral trial tool helps the surgeon to decide on the offset of the femoral base. The selected femoral base is positioned over the femoral $\mathrm{K}$-wire and aligned correctly before being secured to the femur with one cancellous compression screw and three locking screws.

A curved $7-\mathrm{cm}$ incision is performed on the proximal tibia. The pes anserinus is detached from its tibial insertion and then a subfascial tissue tunnel (preferred by the Author), or a standard subcutaneous tunnel, is created from the distal incision towards the femoral one using blunt dissection.

The tibial plate is locked to the absorber and the absorber is placed in the tunnel through the tibial incision. The proximal end of the absorber is then introduced into the femoral base and locked to the base. The correct offset of the tibial base is assessed with fluoroscopy in an anteroposterior projection, ensuring that there is no impingement between the absorber and medial soft tissue structures.

In slight knee flexion, the medial joint space is closed by applying a varus force to the knee. While varus stress is applied, the tibial base is temporarily fixed with two K-wires and then secured to the tibia, again with one cancellous compression screw and three locking screws. The load absorber is then activated by cutting the restraining cable compressing the spring. Before wound closure, it is important to verify that the absorber is deactivated (uncompressed) after approximately $30^{\circ}$ of knee flexion and that no impingement of the device occurs through the full range of motion (ROM). The fascia surrounding the device should not be too tight, and if necessary, should be released (Fig. 2). A Redon-type drain is placed at the end of surgery and left in place for 24-48 hours. Partial weight bearing is allowed from the day after surgery until two weeks postoperatively. Unlimited knee flexion is permitted after surgery as tolerated by the patient.

All 53 patients were discharged from hospital between two and three days after surgery with a specific reha-
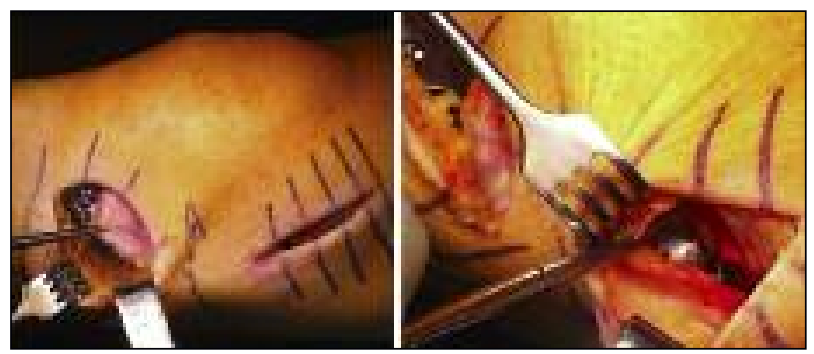

Fig. 2. Subfascial positioning of the KineSpring device.

bilitation protocol and a schedule of follow-up visits. Outcome measurements

The patients returned for follow-up visits at two weeks, one month, three months, six months and one year, and annually thereafter. Each evaluation included a complete clinical and orthopedic examination. Patient self-assessments were performed pre-operatively and at 3, 6, and 12 months postoperatively and included the following scores: KOOS, Tegner activity score, Lysholm functional knee score, VAS pain score and IKDC score. Intraoperative and postoperative adverse events related to the device or the surgical procedure were also evaluated.

\section{Data analysis}

All patients treated with the KineSpring system and having at least three months of postoperative data available were included in this interim analysis. 


\section{p

Descriptive statistics were used to summarize the patient group. Continuous data were reported as mean \pm standard deviation (SD). P-values for changes in patient-reported outcome scores were determined using paired t-test comparison of post-treatment score with baseline. A p-value $<0.05$ was considered statistically significant. Data were analyzed using SAS software. WOMAC domains were calculated from the individual KOOS items: Pain corresponded to KOOS Pain items 5-9, Stiffness to KOOS Symptom items 67, and Function to KOOS ADL (all 17 items).

\section{Results}

The procedure was successfully performed in all the patients: all devices were successfully implanted without intraoperative complications.

Since this is an interim analysis, not all the patients have reached the final follow-up. Moreover, patients who were referred to the hospital and lived further away may not have been able to attend all follow-up visits. The number of patients therefore varies at the different follow-up time points (Tab. 1).

Knee pain and function significantly improved after KineSpring implantation. Mean KOOS subscale scores improved during the follow-up time. All subscale scores were significantly different from the baseline values at 6 and 12 months postoperatively $(\mathrm{p}<0.05)$, except for the Sport/Recreation score at 6 months (Fig. 3). Mean WOMAC Pain and Function domains improved over time and were significantly different compared to baseline at 3, 6 and 12 months $(p<0.05)$. Mean Stiffness was not different from baseline at any postoperative time point (Fig. 4). The mean Lysholm score improved over time and the scores at 3, 6 and 12 months postoperatively were significantly different from baseline $(p<0.05)$ (Fig. 5). The mean IKDC scores at 3, 6 and 12 months postoperatively were signifi-

Table 1. Number of patients $(\mathrm{N})$ available for analysis of patientreported outcome scores.

\begin{tabular}{ll}
\hline Time point & N \\
\hline Baseline & 45 \\
Three-month follow-up & 14 \\
Six-month follow-up & 24 \\
Twelve-month follow-up & 10 \\
\hline
\end{tabular}

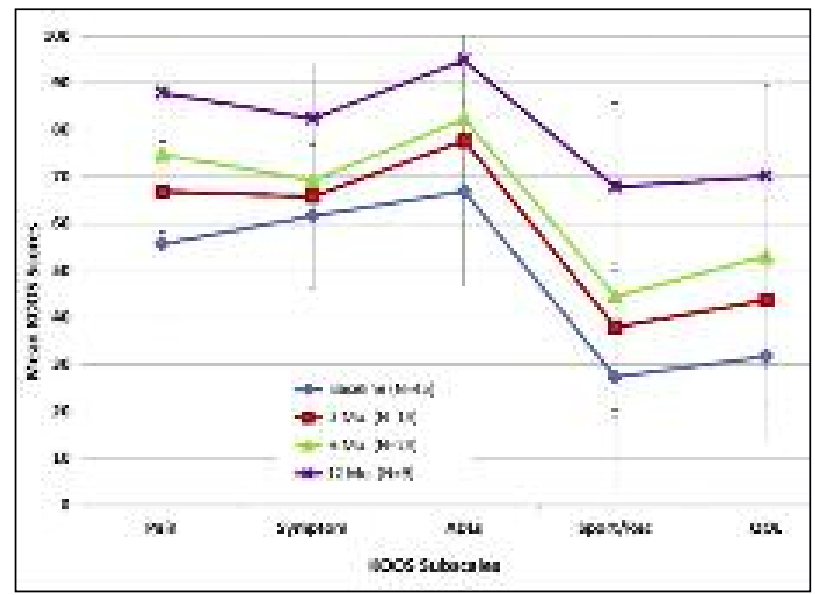

Fig. 3. All KOOS subscales significantly improved compared to baseline values at 6 and 12 months postoperatively $(p<0.05)$, except for the Sport/Recreation score at six months.

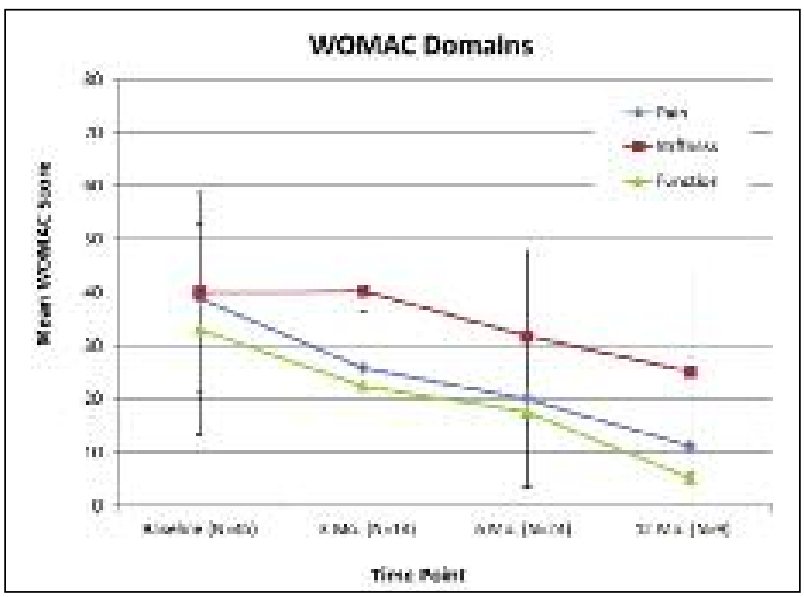

Fig. 4. Mean WOMAC Pain and Function domains significantly improved compared to baseline at three, six and 12 months $(p<0.05)$. Mean Stiffness was not different from baseline at any postoperative time point.

cantly improved compared to baseline $(\mathrm{p}<0.05)$ (Fig. 6). The mean VAS pain score improved over time and the scores at 3, 6 and 12 months postoperatively were significantly different from the baseline score $(\mathrm{p}<0.05)$ (Fig. 7). The mean Tegner score improved slightly over time, however none of the postoperative scores was significantly different from baseline (Fig. 8).

Re-operations were necessary in 5 of the $53(9.4 \%)$ patients. In one case the KineSpring device was removed due to infection. The patient started to present with signs and symptoms of infection two months after surgery. In agreement with an infectivologist, antibiotic therapy with trimethoprim/sulfamethoxa- 


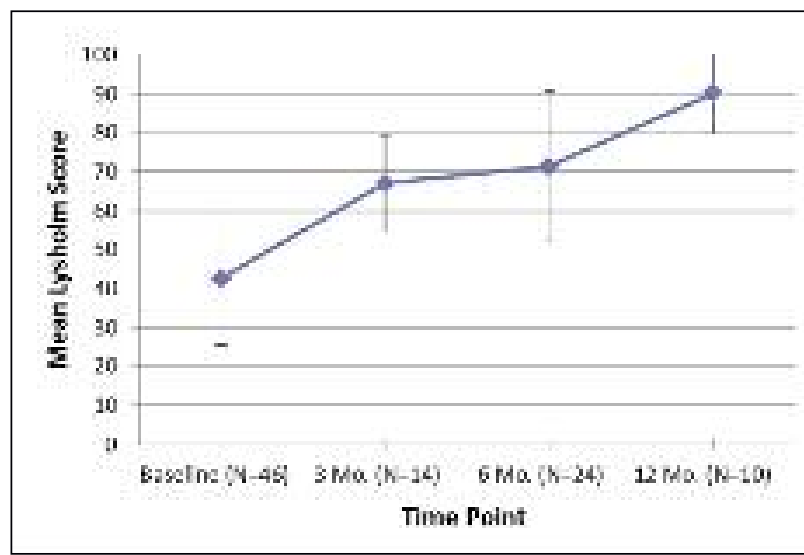

Fig. 5. Mean Lysholm score significantly improved compared to baseline at 3,6 and 12 months $(p<0.05)$.

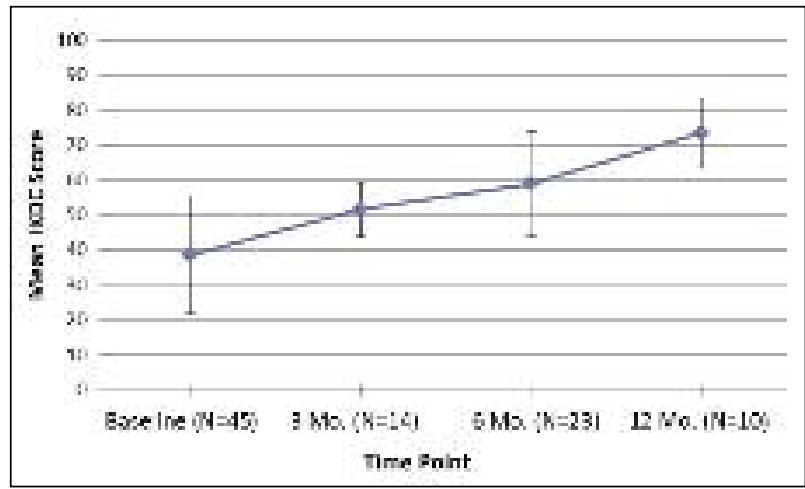

Fig. 6. Mean IKDC score significantly improved compared to baseline at 3,6 and 12 months $(p<0.05)$.

zole and rifampicin was administered for six weeks, leading to partial resolution of the symptoms.

However, after termination of the antibiotic therapy clinical signs and symptoms of infection recurred and the KineSpring system was removed. Two patients presented with pain and stiffness which could not be resolved with conservative therapy. The pain started eight and 10 weeks after surgery respectively; it was located on the medial aspect of the knee. The pain was not related to weight bearing or movement and the patients stated that it was different from the preoperative pain. KineSpring removal resolved the pain. However, six and eight months later the patients started to complain again of the medial OA knee pain they had experienced before KineSpring implantation.

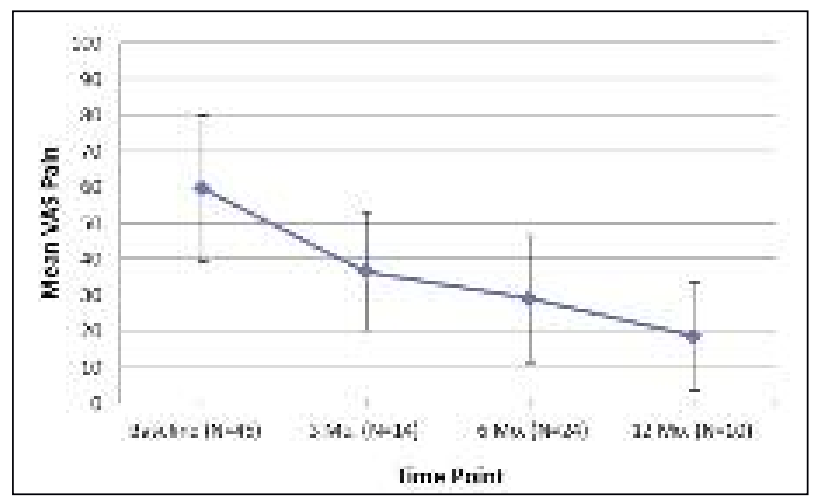

Fig. 7. Mean VAS pain score significantly improved compared to baseline at 3, 6 and 12 months $(p<0.05)$.

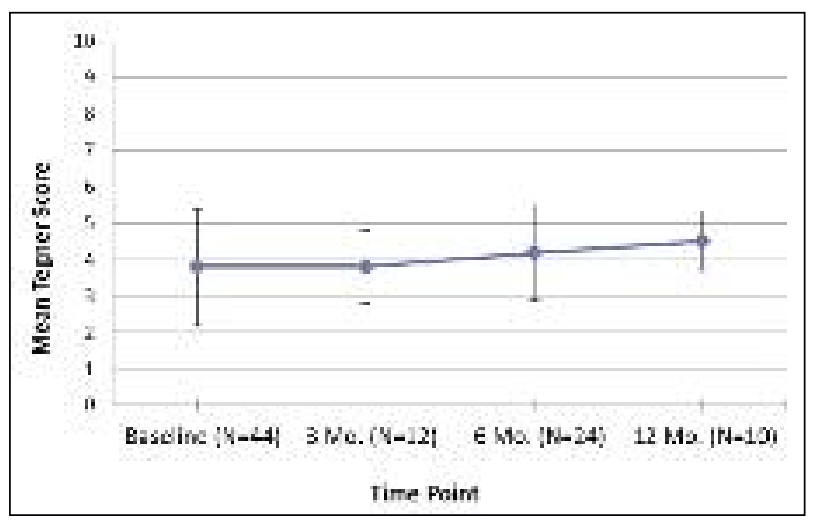

Fig. 8. Mean Tegner score improved slightly over time, however none of the postoperative scores was significantly different from baseline.

Finally, in two cases surgical arthrolysis with release of subcutaneous scar tissue was performed to treat stiffness and limited ROM following implantation. These patients experienced delayed recovery due to problems not related to the procedure and when seen again at follow-up they presented with severe stiffness and flexion contracture which was impossible to treat conservatively. After surgical treatment they regained complete extension and a mean knee flexion of $130^{\circ}$ (range $\left.120-140^{\circ}\right)$. The mean time to re-intervention was 161.8 \pm 85.1 days (range, 92-291).

In the first fifteen patients who underwent KineSpring implantation we observed delayed wound healing of the tibial incision. We thus changed the tibial approach to a curved skin incision in order to avoid stretching 
of the skin during surgery and obtained good results with wound healing. Eight patients complained of pain on the medial aspect of the knee that radiated distally to the patella towards the lateral aspect of the leg. This symptom occurred between two and 10 weeks after surgery. The pain was related to a mid-flexion position of the knee and unrelated to weight bearing. We assumed that it was due to irritation of the infrapatellar branch of the saphenous nerve. Our hypothesis was corroborated by the complete resolution of pain after subcutaneous injection of anaesthetic into the starting point of the pain. All cases were successfully treated with pregabalin $(75 \mathrm{mg}$ twice a day for 5 days, then $150 \mathrm{mg}$ twice a day for 20 days), with pain resolution being achieved between two and three months.

\section{Discussion}

The onset and progression of knee OA are largely caused by biomechanical factors such as excessive mechanical stresses acting across the joint $(10,11)$. Changing the adverse mechanical environment that produced the predisposing conditions for $\mathrm{OA}$ is the key to providing lasting relief from symptoms and to slowing down OA progression (12). It has been demonstrated that after axial correction of a varus knee with HTO, biological changes occur in the chondral tissue of the affected compartment. Load redistribution is able to allow a progressive repair process through the formation of fibrocartilage and remodeling of the subchondral plate, which results to a certain degree in joint space widening and bone remodeling $(13,14)$. Yanai et al. conducted a preclinical study on rabbits and demonstrated that in a full-thickness cartilage defect of the tibial plateau joint distraction alone resulted in significant cartilage repair (15). A gait simulation study on six cadaver knees has demonstrated that KineSpring can successfully unload the medial compartment of the knee (7), but clinical experience with KineSpring for the treatment of knee OA is still limited. London et al. $(8,9)$ reported good short-term results with significant improvement in WOMAC and VAS pain scores in 100 patients at one-year minimum follow-up. Our experience demonstrated that KineSpring is able to provide a significant improvement in knee pain and function in selected patients. WOMAC
Pain and Function domains, as well as IKDC, KOOS and VAS pain scores all showed significant improvement after surgery.

Furthermore, this technique has several advantages over other treatment methods such as HTO or UKA: it is a true tissue-sparing procedure that does not alter the knee biomechanics, and it is readily accepted by patients, given the reversible nature of the procedure. However, it also became evident that the device affects the surrounding soft tissues, causing symptoms that are challenging to diagnose and treat correctly. In our series, two patients suffered medial knee pain that was very difficult to diagnose and to treat and required removal of the implant. Irritation of the infrapatellar branch of the saphenous nerve is a very painful condition that affected $15 \%$ of our patients, causing delayed recovery. In a pre-clinical study on 11 sheep, performed to investigate the tissue response to the KineSpring device, an early inflammatory response around the device was observed four weeks after surgery (16). By twelve weeks after surgery, the inflammatory response was resolved and a pseudosynovial membrane had formed around the absorber. In our series, all the patients who suffered from postoperative pain presented with symptoms between two weeks and three months after surgery. We speculate that the transitory inflammatory response that the device evokes before it becomes "tolerated" by the surrounding soft tissues may be the cause of the early pain. Further histological studies may help to clarify this question. In our experience, KineSpring is a very effective alternative to HTO and UKA. We believe that correct patient selection is the key to obtaining a successful outcome, because the device is designed to relieve weight-bearing knee pain during gait (i.e. pain occurring during the stance phase). Patients who experience pain during squatting or stair climbing will not be satisfied after KineSpring implantation because the device has no function at high flexion angles.

Limitations of this study include the difficulty of interpreting interim data when more baseline data than post-treatment data is available. For this reason, absolute levels of the different outcome scores may be deceptive. Furthermore, in the absence of a control treatment, significant changes from baseline do not necessarily indicate a treatment effect.

In conclusion, on the basis of the very preliminary results of our prospective series, we believe that the 
KineSpring device is able to treat the symptoms of medial compartment $\mathrm{OA}$ in selected patients.

Unloading the medial compartment of the knee may be the key for modifying the adverse mechanical joint environment that triggers the development of $\mathrm{OA}$ and may be able to provide lasting symptom relief and possibly slow down the progression of the pathology. Further studies are necessary to better understand the true therapeutic potential of this novel device.

\section{References}

1. Zhang Y, Jordan JM. Epidemiology of osteoarthritis. Clin Geriatr Med. 2010;26:355-369.

2. Ledingham J, Regan M, Jones A, et al. Radiographic patterns and associations of osteoarthritis of the knee in patients referred to hospital. Ann Rheum Dis. 1993;52:520-526.

3. Koo S, Andriacchi TP. A comparison of the influence of global functional loads vs. local contact anatomy on articular cartilage thickness at the knee. J Biomech. 2007;40:2961-2966.

4. Thorp LE, Sumner DR, Wimmer MA, et al. Relationship between pain and medial knee joint loading in mild radiographic knee osteoarthritis. Arthritis Rheum. 2007;57:1254-1260.

5. Crawford DC, Miller LE, Block JE. Conservative management of symptomatic knee osteoarthritis: a flawed strategy? Orthop Rev. (Pavia) 2013;5:e2

6. Niinimäki T, Eskelinen A, Mäkelä K, et al. Unicompartmental knee arthroplasty survivorship is lower than TKA survivors- hip: a 27-year Finnish registry study. Clin Orthop Relat Res. 2014;472:1496-1501.

7. Gabriel SM, Clifford AG, Maloney WJ, et al. Unloading the osteoarthritic knee with a novel implant system. J Appl Biomech. 2013;29:647-654.

8. London NJ, Smith J, Miller LE, et al. Bridging the osteoarthritis treatment gap with the Kine Spring Knee Implant System: early evidence in 100 patients with 1 -year minimum follow-up. Orthopedic Research and Reviews. 2013;5:65-73.

9. London NJ, Smith J, Miller LE, et al. Midterm outcomes and predictors of clinical success with the KineSpring Knee implant system. Clin Med Insights Arthritis Musculoskelet Disord. 2013;6:19-28.

10. Radin EL. Who gets osteoarthritis and why? An update. J Rheumatol. 2005;32:1136-1138.

11. Block JA, Shakoor N. The biomechanics of osteoarthritis: implications for therapy. Curr Rheumatol Rep. 2009;11:15-22.

12. Brandt KD, Dieppe P, Radin E. Etiopathogenesis of osteoarthritis. Med Clin North Am. 2009;93:1-24.

13. Radin EL, Burr DB. Hypothesis: joints can heal. Semin Arthritis Rheum. 1984;13:293-302.

14. Koshino T, Wada S, Ara Y, et al. Regeneration of degenerated articular cartilage after high tibial valgus osteotomy for medial compartmental osteoarthritis of the knee. Knee. 2003; 10: 229-236.

15. Yanai T, Ishii T, Chang F, et al. Repair of large full-thickness articular cartilage defects in the rabbit: the effects of joint distraction and autologous bone-marrow-derived mesenchymal cell transplantation. J Bone Joint Surg Br. 2005;87:721-729.

16. Allen MJ, Townsend KL, Bauer TW, et al. Evaluation of the safety of a novel knee load-bypassing device in a sheep model. J Bone Joint Surg Am. 2012;94:77-84. 